\title{
Effective FM Bandwidth Estimate Scheme with the DARC in Broadcasting Networks
}

\author{
Sang Woon Lee ${ }^{1}$, Kyoo Jin $\mathrm{Han}^{2}$, Keum Chan Whang ${ }^{3}$ \\ ${ }^{1}$ Senior Researcher, Technical R\&D Center, MBC, Korea \\ 1sw@mbc.co.kr \\ ${ }^{2}$ Senior Researcher, CDMA System Lab. LG Electronics, Korea \\ ${ }^{3}$ Dept. of Electrical and Electronics Eng. Yonsei Univ., Korea
}

\begin{abstract}
In this paper we propose the effect on the RF bandwidth when the DARC data signal is added to the ordinary FM broadcasting signal. Generally, the bandwidth of the commercial FM broadcasting signal is strictly restricted to $200 \mathrm{KHz}$. Hence, even though the DARC data signal is added at the ordinary stereophonic FM signal, the required bandwidth should not exceed $200 \mathrm{KHz}$. The simulation results show that even in the worst case, the required bandwidth is about $184 \mathrm{KHz}$, and the rest of $16 \mathrm{KHz}$ bandwidth could be used for other FM data broadcasting services.
\end{abstract}

\section{Introduction}

After the service of the FM multiplex broadcasting called DARC was started at the mid of 1990, it has been much interested in the mobile data broadcasting service. By virtue of the broadcasting characteristics of the FM audio service, DARC system makes the information data broadcasting service to the many customers distributed in the wide area to be possible. Service area has been also widely extended from the traffic, DGPS (Differential Global Positioning System), weather, news to ITS (Intelligent Transportation System), Telematics [1,2].

The detail specification of DARC system is known by ITU-R [3], and the performance analyses of the DARC system on the several constituent parts such as level-controlled MSK, the immunity on the multi-path fading environments, or error correction ability have been carried out at the papers $[4,5]$.

RF bandwidth of the commercial FM broadcasting signals is usually set to $200 \mathrm{KHz}$. Even though it is known that DARC service is possible within the bandwidth for the FM broadcasting service, there has been no work in which precise analysis on the RF bandwidth of the DARC system is treated. And this work is useful in the aspect of the efficient usage of the valuable frequency resources.

In this paper we analyze the effect on the RF bandwidth when DARC data signal is added to the ordinary FM broadcasting signals. It is performed by the computer simulation in which two systems are compared; one is ordinary FM broadcasting system and the other is the DARC system. Level controller and band-pass filter that are considered in this paper meet the requirement described in the DARC specification [3]. 


\section{DARC System Model}

We fully follow the specification of the FM system and DARC system [3] in order to estimate precisely the RF bandwidth of the ordinary FM broadcasting system and the FM system including DARC data. Fig.1 shows the block diagram of the DARC system considered in this paper. The system consists of the stereophonic matrix containing $\mathrm{L}+\mathrm{R}$ and $\mathrm{L}-\mathrm{R}$ signals, $19 \mathrm{KHz}$ pilot generator, frequency multiplier (X2 and $\mathrm{X} 4$ ), data signal generator, FM generator, and the modulation level is controlled according to the magnitude of the L-R signal.

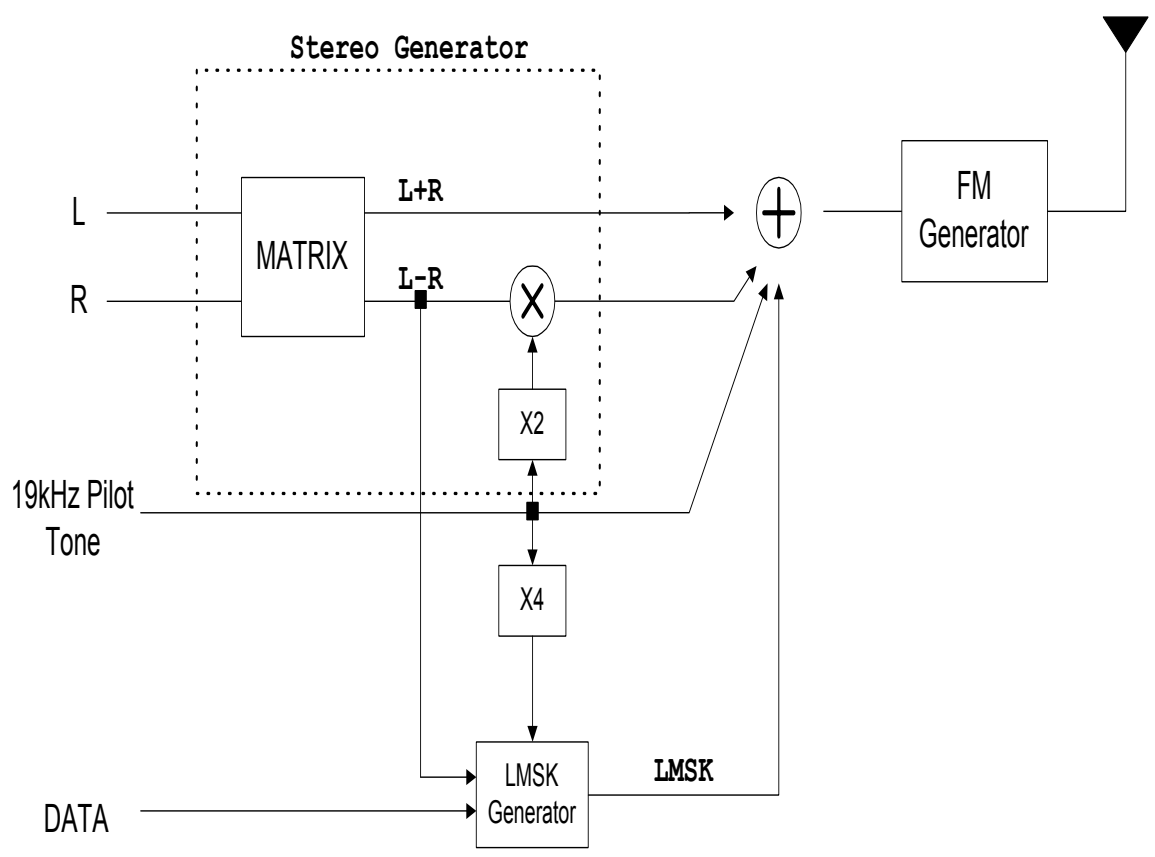

Fig. 1 The block diagram of the DARC system

The inputs of the stereo signal generator are $\mathrm{L}$ channel and $\mathrm{R}$ channel audio signals. At the matrix block, $\mathrm{L}+\mathrm{R}$ signal that is sum of the two audio signals and L-R signal that is the difference between the two signals are generated. Then, L-R signal is frequency shifted by multiplying $38 \mathrm{KHz}$ single-tone carrier.

LMSK generator controls the magnitude of the DARC data signal according to the magnitude of the L-R signal. Its output is MSK modulated and band-pass filtered. The frequency of the sub-carrier for MSK modulation is $76 \mathrm{KHz}$. In the specification of the DARC system [3], the upper bound and lower bound of the frequency response of the DARC data signals are described, which is shown at Fig. 2. To meet the requirement of the frequency response, we have adopted Chebyshef type-2 filter with order 8 [7], whose filter coefficients are chosen as shown in table 1. 


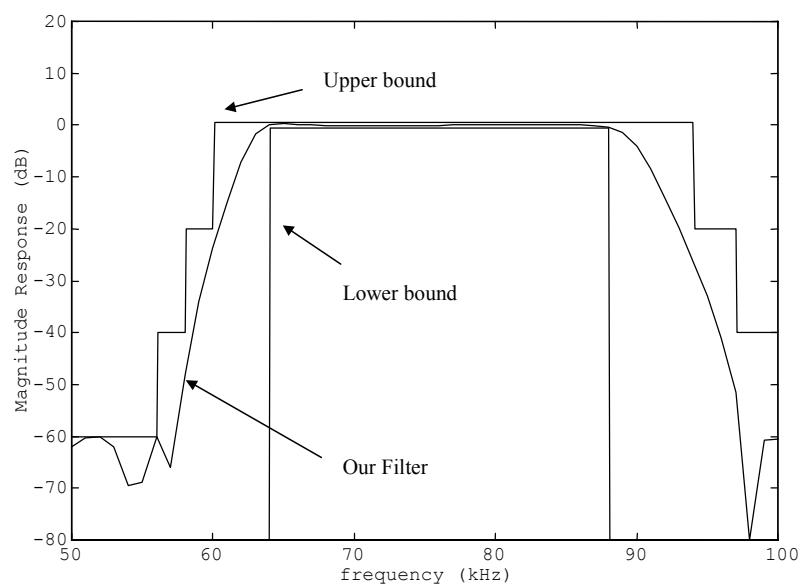

Fig. 2 Filter requirement of the DARC system and the Chebyshef type-2 filter

Table 1 The coefficients of the proposed Chebyshef type-2 filter

\begin{tabular}{|c|c|}
\hline Numerator Coefficients & Denominator Coefficients \\
\hline 0.0009641 & 1 \\
\hline-0.012423622 & -13.40536282 \\
\hline 0.077007238 & 85.71009262 \\
\hline-0.305025502 & -346.663852 \\
\hline 0.865038284 & 992.139065 \\
\hline-1.8644737 & -2129.490727 \\
\hline 3.162690668 & 3544.666673 \\
\hline-4.310679576 & -4666.626359 \\
\hline 4.773804224 & 4910.109636 \\
\hline-4.310679576 & -4142.52789 \\
\hline 3.162690668 & 2793.189761 \\
\hline-1.8644737 & -1489.580883 \\
\hline 0.865038284 & 616.0639585 \\
\hline-0.305025502 & -191.086542 \\
\hline 0.0009641 & 0.38564381 \\
\hline
\end{tabular}




\section{Simulation and Results}

\subsection{The Characteristics of the Base Band Signal}

We explore the effects on the frequency responses of the $\mathrm{L}+\mathrm{R}$ and L-R signals according to the correlation of the $\mathrm{L}$ and $\mathrm{R}$ channel signals. The correlation coefficient $c f$ of the input signals is defined as follows.

$$
c f=\frac{\operatorname{cov}(L, R)}{\sqrt{\operatorname{cov}(L, L) \cdot \operatorname{cov}(R, R)}}
$$

Here, Cov is the covariance, and L and R is channel audio signals, respectively. Because the DARC system adopts the LMSK modulation method, in which the injection level is controlled by the magnitude of the L-R signal, the level is adjusted according to the correlation coefficient of the $\mathrm{L}$ and $\mathrm{R}$ channel signals. Generally, the bandwidth of the $\mathrm{L}$ and $\mathrm{R}$ channel signals for FM broadcasting should not exceed $15 \mathrm{KHz}$, so two stereophonic signals whose bandwidths are $15 \mathrm{KHz}$ are generated. Table 2 shows the relation between the correlation coefficients and the average injection level, where the following two input cases are considered. First, the correlation of the two stereophonic signals is 0.9 , and second, the correlation is zero so that the two audio input signals are independent.

Fig. 3 shows, by base-band frequency spectrum, the relation between the magnitudes of the LMSK modulated DARC signals and the correlation coefficient between the L and R channel input signals. As the correlation coefficient is higher, the magnitude of the frequency spectrum of the L-R signals is also increased.

Table 2 The correlation coefficient and the average injection level of the DARC signals

\begin{tabular}{cc}
\hline$c f$ & Average Injection Level for DARC \\
\hline 0.9 & $6.67 \%$ \\
0 & $8.91 \%$ \\
\hline
\end{tabular}

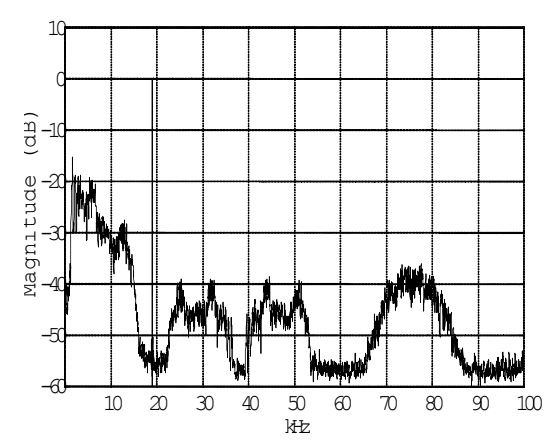

(a)The cf of the $\mathrm{L}$, R channel signals is 0.9

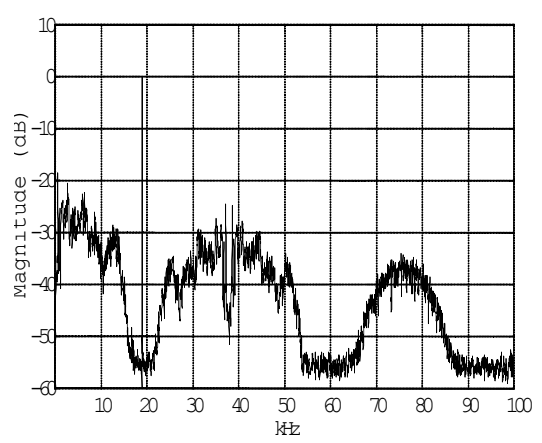

(b) The cf of the L, R channel signals is zero

Fig. 3 Base-band frequency spectrums of the ordinary FM signals and DARC signal 


\subsection{FM Bandwidth in the Stereophonic Signals}

The bandwidth of a signal can be defined that the bandwidth in which $99 \%$ of the total power spectrum of the signal is contained [8]. In this paper, 4 cases are considered and the detail simulation conditions are described as follows.

Case 1: the correlation coefficient of the $\mathrm{L}$ and $\mathrm{R}$ channel signals is 0.9 .

Case 2: the correlation coefficient of the Land $\mathrm{R}$ channel signals is zero.

Case 3: $\mathrm{L}$ and $\mathrm{R}$ channel signals are independent of each other but their most powers are concentrated within the bandwidth between $10 \mathrm{KHz}$ and $15 \mathrm{KHz}$.

Case 4: Both of the $\mathrm{L}$ and $\mathrm{R}$ channel signals are $15 \mathrm{KHz}$ single tones with random phase.

At Table 3, the estimated RF bandwidths according to the above four input conditions are present. From the results, it is known that the case 4 requires the largest bandwidth and the required bandwidth gets smaller as the correlation coefficient between $\mathrm{L}$ and $\mathrm{R}$ signals increases.

Fig. 4 shows the frequency spectrum when the maximum frequency deviation $\Delta f$ is set to $75 \mathrm{KHz}$ and the stereophonic FM input signals fall under one of following three conditions; case 1,2, and 4. The graphs show that among them the 4(c) requires largest bandwidth and 4(a) occupies minimum bandwidth.

As mentioned at the above section, because the magnitude of the L-R signal is frequency shifted by a $38 \mathrm{KHz}$ carrier, frequency shifted L-R signals put more effect on the FM modulated total bandwidth than L+R signals do. Hence FM modulated total bandwidth get larger as the magnitude of the L-R is larger.

Table 3 The required frequency bandwidths of the ordinary FM system

\begin{tabular}{lc}
\hline & Bandwidth $(\mathrm{KHz})$ \\
\hline Case $1, c f=0.9$ & 84.6 \\
Case $2, c f=0$ & 93.8 \\
Case 3, 10-15KHz & 147 \\
Case 4, $15 \mathrm{KHz}$ & 182 \\
\hline
\end{tabular}

\subsection{FM Bandwidth with the DARC Data Signal}

Fig. 5 shows the RF spectrums under the condition of the input sources are same as the case of FIg. 4 when the maximum frequency deviation, $\Delta f$, is $75 \mathrm{KHz}$ and the DARC data signal is added to the stereophonic FM signal. From the graphs, we can see that case 3 gets the maximum frequency bandwidth of $184 \mathrm{KHz}$. 


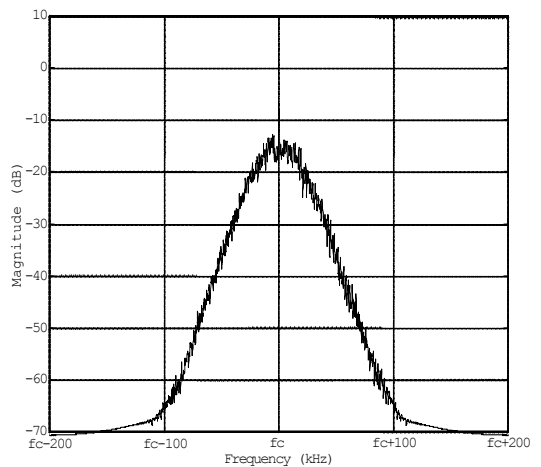

(a) The $\mathrm{cf}$ of the $\mathrm{L}, \mathrm{R}$ channel signals is 0.9

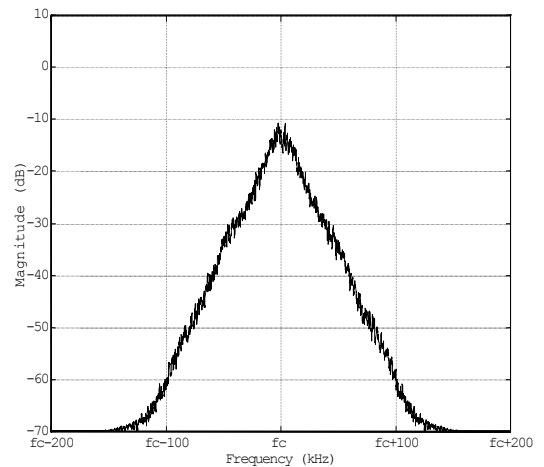

(b)The cf of the L, R channel signals is zero

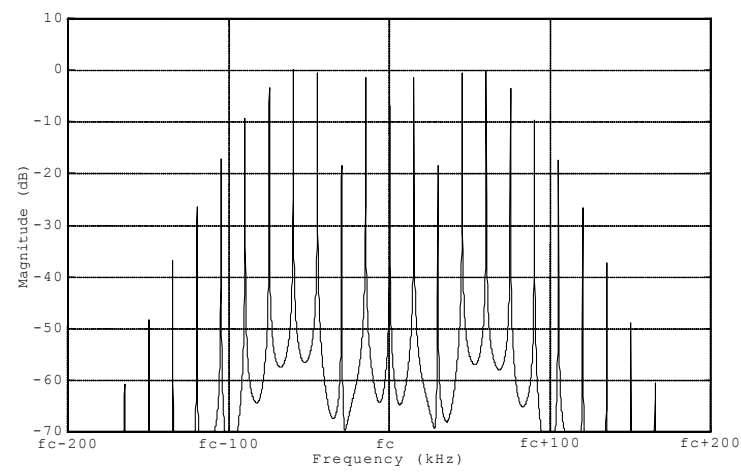

(c) Both of the $\mathrm{L}$ and $\mathrm{R}$ channel signals are $15 \mathrm{KHz}$ single tones

Fig. 4 Frequency spectrums of the ordinary FM systems

\section{Conclusions}

In this paper we analyze the effect on the RF bandwidth when the DARC data is added to FM broadcasting signals. As mentioned above, the FM broadcasting system and DARC data adding mechanism that is presented in this paper fully follows the ITU-R specification. Our research is on that how much the RF bandwidths of the ordinary FM broadcasting system and DARC system are affected by the statistics of the stereophonic input signals for FM broadcasting. The results show that, in the worst case, RF bandwidths of the ordinary FM broadcasting system and the DARC system are $182 \mathrm{KHz}$ and $184 \mathrm{KHz}$, respectively. It means that in the ordinary FM broadcasting system there is still enough frequency space for the additional data services. Moreover the total RF bandwidth does not exceed $200 \mathrm{KHz}$, that is the requirement of the FM broadcasting service, even though DARC data signal is added to the ordinary FM broadcasting signals. It can be useful baseline results for the efficient usage of the frequency resources such as additional data broadcasting service in the ordinary FM frequency band. 


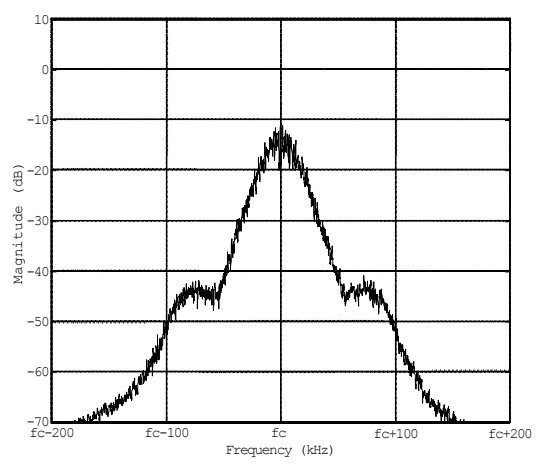

(a) The cf of the $\mathrm{L}$, $\mathrm{R}$ channel signals is 0.9

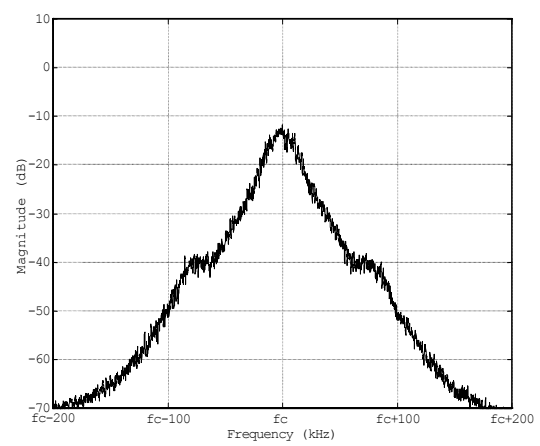

(b) The cf of the L, R channel signals is zero

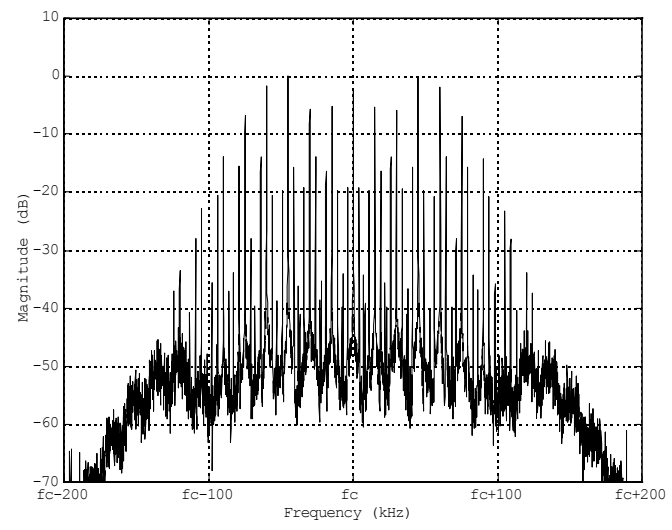

(c) Both of the $\mathrm{L}$ and $\mathrm{R}$ channel signals are $15 \mathrm{KHz}$ single tones

Fig. 5 Frequency spectrums when the DARC data signals are added at the ordinary FM signals

\section{References}

[1] Chen Wei and Shen-Weng Hong, An FM Sub-carrier Data Broadcasting System On Bus Transport Indication System, IEEE Transactions on Broadcasting, Vol. 47. No.1 Mar. 2001

[2] Jonguk Park, Jeongho Joh, Hyungchul Lim, Pilho Park and Sangwoon Lee, "THE DEVELOPMENT OF DGPS SERVICE SYSTEM USING FM DARC IN KOREA", Proceedings on the Federation of International Federation of Surveyors, May 2001

[3] ITU-R BS Recommendation DOC. 1194, 1995.

[4] Marion J. de Ridder-de Groote and Ramjee Prasad, Jan. H.Born, "Analysis of New Methods for Broadcasting Digital Data to Mobile Terminal over an FM-Channel”, IEEE Transactions on Broadcasting, Vol. 40. No. 1. Mar. 1994

[5] K. Kuroda and M Takada, T. Isobe, "Transmission Scheme of of High-capacity FM Multiplex Broadcasting System", IEEE Transactions on Broadcasting, Vol. 42. No.3 Sep. 1996 
[6] Papoulis, Probability, Random Variables, and Stochastic Processes. Third Edition, McGraw Hill, 1991.

[7] Michel C. Jeruchim, Philip Balaban, and K. Sam Shanmugan, Simulation of Communication Systems, Plenum Press, 1992.

[8] E B. Crutchfield, NAB Engineering Handbook 7th edition, NAB, 1985.

[9] ITU-R BS Recommendation DOC. 643, 1986

[10] Ferrel G. Stremler, Introduction to Communication Systems, Third Edition, AddisonWesley, 1990.

[11] J. R. Carson, "Notes on the Theory of Modulation," Proceedings of the IEEE, Vol. 51 (1951) : pp 893-896. 